\title{
COMPARATIVE ANALYSIS OF VARIANTS OF GABOR-WIGNER TRANSFORM FOR CROSS-TERM REDUCTION
}

\author{
Muhammad Ajab"), Imtiaz Ahmad Taj ${ }^{1)}$, Nabeel Ali Khan ${ }^{2)}$ \\ 1) Muhammad Ali Jinnah University (MAJU), Islamabad, Pakistan ( $\triangle$ ajabatcop@yahoo.com) \\ 2) Federal Urdu University (FUUAST), Islamabad, Pakistan
}

\begin{abstract}
Gabor Wigner Transform (GWT) is a composition of two time-frequency planes (Gabor Transform (GT) and Wigner Distribution (WD)), and hence GWT takes the advantages of both transforms (high resolution of WD and cross-terms free GT). In multi-component signal analysis where GWT fails to extract auto-components, the marriage of signal processing and image processing techniques proved their potential to extract autocomponents. The proposed algorithm maintained the resolution of auto-components. This work also shows that the Fractional Fourier Transform (FRFT) domain is a powerful tool for signal analysis. Performance analysis of modified fractional GWT reveals that it provides a solution of cross-terms of WD and blurring of GT.
\end{abstract}

Keywords: Wigner Distribution, Gabor Transform, Gabor Wigner Transform, Fractional Fourier Transform.

(C) 2012 Polish Academy of Sciences. All rights reserved

\section{Introduction}

Time representation of a one-dimensional signal lacks the frequency description of the signal. On the other hand, the Fourier Transform (FT) of the signal cannot describe how the spectral content of the signal alters with respect to time. Therefore a time variable is induced in FT to obtained information about the changes of spectral content of a signal with respect to time. Hence, the basic goal of a time-frequency representation (TFR) is to find out the energy concentration along the frequency axis at a given time $[1,2]$.

A TFR is a two-dimensional function which provides simultaneously, temporal and spectral information and therefore is used to analyze the non-stationary signals (seismic signals, radar signals, voice communication signals, biomedical signals, etc.). It provides the information which is unavailable in time or frequency representation alone. Time frequency representations (TFRs) provide information such as auto-components (liner chirp, quadratic chirp, sinusoid and Gaussian atom etc.) in the signal, their time duration and frequency bands over which these components are defined and their relative amplitudes [3, 4].

TFRs are classified as Linear TFRs and Quadratic TFRs. Linear TFRs (short time Fourier Transform, Gabor Transform (GT), wavelet transform, etc.) obey the principle of superposition. Linear TFRs offer no cross-terms but have low resolution of autocomponents. Quadratic TFRs (Wigner Distribution (WD), S-method, etc.) offer better resolution of auto-components but have cross-terms $[1,5]$. This discussion shows that there is no unique TFR that tackles all possible applications. The choice of a particular TFR depends on specific application at hand. However, TFRs have proved themselves to be the key to successful extraction and classification of signals in numerous applications. TFRs are often compared in terms of their ability to suppress cross-terms, resolution performance and mathematical properties [4]. 
WD is the most popular quadratic TFR $[6,7]$ and shows its high resolution property for analysis of a linear chirp signal and a Gaussian atom, while in case of a quadratic component and multiple components, WD has a cross terms problem $[5,8]$. Nice mathematical properties of the WD like infinite time and frequency resolutions (there is no window in (3)), preservation of time and frequency support and more, made WD a powerful tool for analysis of signals [7,9]. Similarly WD has the drawback of its quadratic nature and introduces the cross-terms which makes difficult to visualize the time-frequency plane [10]. Different variants of WD were proposed to eliminate its cross-terms [11, 12].

A non-linear filtering technique was proposed in [13] to reduce cross-terms of WD. This technique is based on intuition that auto-terms dominant regions have significantly less variation as compared to cross-terms dominant regions. Thus in regions of low variation the proposed nonlinear filter converts itself close to identity operator while in regions of crossterms it becomes a low pass filter. This non-linear filter performs better than kernel-based techniques but in regions where cross-terms overlap auto-components this technique fails to give optimum results.

Fractional Fourier Transform (FRFT) and signal synthesis based recursive technique to eliminate cross-terms of WD without affecting auto-components resolution were proposed in [14]. This method performs well in highly challenging situations i.e. when auto-components overlap cross-terms. This technique exploits the mismatch of fractionally rotated and aligned back WDs to detect cross-terms. The iterative nature of this method limits its scope for parallel implementation and also its computational cost is high.

Image processing techniques and FRFT can be used for cross-terms suppression of WD [15]. This technique uses the support vector machine for classification of auto-components. Auto-components of a multi-component signal are isolated in the FRFT domain. The main drawback of this method is auto-component's discontinuity due to image segmentation.

A non-linear morphological filters based technique to eliminate cross-terms of WD was proposed in [16]. In this method a marker is obtained through a spectrogram which is used for morphological analysis of WD. This technique preserves auto-component's resolution. This non-linear morphological filter fails to give optimum results where auto-components overlap cross-terms.

The main feature of WD is its high resolution property of auto-components during the analysis of mono-component signals. But WD has cross-terms in case of quadratic and multicomponent signals. The main feature of the GT is its linearity property but it has less resolution of auto-components than WD. For achieving the goals such as (i) high concentration of auto-components and (ii) elimination of the cross-terms, it is necessary to combine excellent features of both WD and GT [17-19].

In this research, a modified fractional GWT has been developed by marriage of signal processing and image processing techniques. The proposed method is applied to analyze multi-component signal's components extraction and to tackle the resolution problem faced by GT. This work has shown that modified fractional GWT combines the advantages of the GT and the WD. Performance analysis shows that the proposed method has high resolution of auto-components as compared with other TFRs.

The paper is organized as follows. The theoretical background relevant to the present work is presented in Section 2 and Section 3. An algorithm for modified fractional GWT is described in Section 4. Performance analysis of modified fractional GWT is described in Section 5. Section 6 concludes the paper. 


\section{Gabor Wigner Transform}

Short time Fourier Transform (STFT) [5, 6], the simplest TFR, has been introduced for better time localization of the frequency contents of a signal by using a suitable window. By using the STFT we can observe how the frequency of the signal changes with time. It multiples the signal with a symmetric sliding window, $w(t-\tau)$ and then transforms it to the frequency domain. The STFT of a signal $x(t)$ is defined as:

$$
\operatorname{STFT}_{x}(t, \omega)=\int_{-\infty}^{\infty} x(\tau) w(t-\tau) \exp (-j \omega \tau) d \tau .
$$

In implementation of STFT the choice of the window function is very important. A wide window provides high frequency resolution while a narrow window has high time resolution. Once the analysis window has been chosen, the resolution is set for both time and frequency. We cannot have a function that has an arbitrary short time duration and narrow frequency bandwidth at the same time. According to Uncertainty principle it is impossible to get both frequency and time resolution at the same time [20]. However the Gaussian window achieves an optimal joint time-frequency concentration with maximum possible resolution in both domains.

The STFT with Gaussian window is called a Gabor Transform [17, 21]. The main feature of the GT is linearity but GT has less resolution of auto-components than WD. The GT does not have the cross-term problem. Mathematically GT of $x(t)$ is defined as,

$$
G T_{x}(t, \omega)=\sqrt{\frac{1}{2 \pi}} \int_{-\infty}^{\infty} e^{-(\tau-t)^{2} / 2} e^{-j \omega(\tau-t / 2)} x(\tau) d(\tau) .
$$

WD is the most popular TFR [6,7]. Mathematically, WD of a signal $x(t)$ is defined as:

$$
W D_{x}(t, \omega)=\int_{-\infty}^{\infty} x(t+\tau / 2) x^{*}(t-\tau / 2) e^{-j \omega \tau} d \tau
$$

where $x^{*}(t)$ is the complex conjugate of $x(t)$. WD is not linear since

$$
W D_{x}(t, \omega) \neq W D_{x_{1}}(t, \omega)+W D_{x_{2}}(t, \omega),
$$

where

$$
x(t)=x_{1}(t)+x_{2}(t) .
$$

Consider a signal $x(t)$ which consists of $M$ auto-components

The WD of $x(t)$ is given by

$$
x(t)=\sum_{i=1}^{M} x_{i}(t) .
$$

$$
W D_{x}(t, \omega)=\sum_{i=1}^{M} W D_{x_{i}}(t, \omega)+\sum_{k=1}^{M-1} \sum_{l=k+1}^{M} 2 \operatorname{Re}\left(\left(W D_{x_{k} x_{l}}(t, \omega)\right) .\right.
$$


Equation (7) shows that the WD of the multi-component signal $x(t)$ has $\mathrm{M}$ autocomponents and $M(M-1) / 2$ cross-components. The properties of cross- terms are defined in [22].

The combination of GT and WD is called a Gabor Wigner Transform (GWT) [17-19]. Mathematically:

$$
\begin{gathered}
G W T_{x}(t, \omega)=G T_{x}(t, \omega) W D_{x}(t, \omega), \\
G W T_{x}(t, \omega)=\min \left\{\left|G T_{x}(t, \omega)\right|^{2},\left|W D_{x}(t, \omega)\right|\right\}, \\
G W T_{x}(t, \omega)=W D_{x}(t, \omega)\left\{\left|G T_{x}(t, \omega)\right|>0.25\right\}, \\
G W T_{x}(t, \omega)=G T_{x}^{2.6}(t, \omega) W D_{x}^{0.6}(t, \omega) .
\end{gathered}
$$

Equations $(8,9,10$ and 11) show that there is no unique definition of GWT and choice of GT and WD is critical in order to extract strengths of GT and WD. However different combinations of GWT are only applicable for slowly time-varying signals but fail in case of amplitude-varying time signals as shown in examples 1 and 2.

\section{Fractional Fourier Transform}

FT is one of widely used tools in signal processing [21, 23]. The Fractional Fourier Transform (FRFT) was introduced in [24] and is a generalization of FT. FRFT has established itself as a powerful tool for the analysis of time-varying signals in a very short span of time [25]. FRFT has many applications in filter design, pattern recognition, communication [16, 26, 27] and TFRs [12], etc. FRFT can be used in applications where FT fails to work and its cost of implementation is also low. Windows can be analyzed using FRFT [27]. FRFT can be used for detection of cross terns in WD [14]. FRFT can isolate signal components from a multi-component signal [15]. Mathematically, FRFT [26] of a signal $x(t)$ is defined as

$$
X_{\alpha}(u)=\int_{-\infty}^{\infty} K(\alpha, t, u) x(t) d t
$$

where $\alpha=a \pi / 2$ and $K(\alpha, t, u)$ is a kernel function defined as

$$
K(\alpha, t, u)=\frac{e^{j \alpha / 2}}{\sqrt{j \sin \alpha}} e^{j \pi} \frac{\left(t^{2}+u^{2}\right) \cos \alpha-2 t u}{\sin \alpha} .
$$

Following are the most important characteristics of FRFT :

1. FRFT becomes FT when $\alpha=\pi / 2$.

2. FRFT with $\alpha=0$ or $\alpha=2 \pi$ is equal to an identity operation.

3. $(0<\alpha<2 \pi)$ corresponds to rotation of the time-frequency plane.

4. FRFT is linear, commutative and associative.

In signal analysis a wider window is used to filter a pure sinusoidal signal, while on the other hand to filter a delta pulse a narrower window is needed. When the signal does not match the time or the frequency direction, we rotate the time-frequency plane by taking the FRFT of the signal. The reason of this signal rotation is to find out an appropriate concentration of auto-components [28]. 
The rotation of the time-frequency plane by angle $\alpha$ in transformed coordinates $(u, v)$ is described as:

$$
\left(\begin{array}{l}
t \\
\omega
\end{array}\right)=\left(\begin{array}{cc}
\cos \alpha & -\sin \alpha \\
\sin \alpha & \cos \alpha
\end{array}\right)\left(\begin{array}{l}
u \\
v
\end{array}\right) .
$$

Now the relationship for the FRFT kernel:

$$
K\left(\alpha, t_{o}, u-u_{o}\right) e^{j 2 \pi u_{o} v} e^{-j \pi u v}=\left[K\left(-\alpha, u_{o}, t-t_{o}\right) e^{j \omega t_{o}} e^{-j \frac{\omega}{2} t}\right]^{*} .
$$

Clockwise rotation of WD, GT and GWT is equal to FRFT of these distributions [3, 20]. Mathematically:

$$
\begin{gathered}
W D_{x_{\alpha}}(u, v)=W D_{x}(u \cos \alpha-v \sin \alpha, u \sin \alpha+v \cos \alpha), \\
G T_{x_{\alpha}}(u, v)=G T_{x}(u \cos \alpha-v \sin \alpha, u \sin \alpha+v \cos \alpha), \\
G W T_{x_{\alpha}}(u, v)=G W T_{x}(u \cos \alpha-v \sin \alpha, u \sin \alpha+v \cos \alpha) .
\end{gathered}
$$

Equations $(16,17$ and 18) show that FRFT has a relation with GWT, WD and GT. FRFT, to design a filter [17], is given as:

$$
r(t)=O_{X}^{-\alpha}\left\{O_{X}^{\alpha}[x(t)] H(u)\right\},
$$

where $x(t)$ and $r(t)$ are the filter's input and the output, and $H(u)$ is the transfer function. For more than one $\alpha$, (19) can be written as

$$
\begin{gathered}
x_{1}(t)=O_{X}^{-\alpha_{1}}\left\{O_{X}^{\alpha}[x(t)] H_{1}(u)\right\}, \\
x_{2}(t)=O_{X}^{-\alpha_{2}}\left\{O_{X}^{\alpha_{2}}\left[x_{1}(t)\right] H_{2}(u)\right\}, \\
x_{n-1}(t)=O_{X}^{-\alpha_{n-1}}\left\{O_{X}{ }^{\alpha_{n-1}}\left[x_{n-2}(t)\right] H_{n-1}(u)\right\}, \\
r(t)=O_{X}^{-\alpha_{n}}\left\{O_{X}^{\alpha_{n}}\left[x_{n-1}(t)\right] H_{n}(u)\right\} .
\end{gathered}
$$

Equation (20) tells that the choice of $\alpha_{n}$ is critical in FRFT domain for successful elimination of noise.

To design a filter in the fractional domain, two important parameters are:

1) Rotation angle $\alpha[15,17]$.

2) Selection criteria for cutoff lines $[15,17]$.

\section{Modified Fractional GWT}

The objective of this work is to propose a TFR which should preserve the quality of autocomponents for multi-component dynamic signals and suppress the cross-terms. For this purpose a proper combination of linear and quadratic TFRs such as GT and WD is designed 
which can achieve better results as compared to already proposed definitions of GWT. Steps of the proposed method are given as:

Step 1. Transform the given signal $x(t)$ in 2D using (2).

Step 2. Adaptive thresholding and image segmentation (8-connectivity criterion) of the process performed in step 1 [29]. The steps for adaptive thresholding are

(i). Compute $G T_{x}(t, \omega)$ of the signal $x(t)$ and its mean value $T$, where

$$
T=\text { mean of } G T_{x}(t, \omega) .
$$

(ii). Classify the transformed data into two classes $G T_{A}(t, \omega)$ and $G T_{B}(t, \omega)$ as

$$
\begin{gathered}
G T_{A}(t, \omega) \in G T_{x}(t, \omega) \quad \text { if } \quad G T_{x}(t, \omega) \geq T, \\
G T_{B}(t, \omega) \in G T_{x}(t, \omega) \quad \text { if } \quad G T_{x}(t, \omega)<T .
\end{gathered}
$$

(iii). Compute averages of $G T_{A}(t, \omega)$ and $G T_{B}(t, \omega)$ and update $T$ as

$$
T=\frac{\mu_{G T_{A}(t, \omega)}+\mu_{G T_{B}(t, \omega)}}{2} .
$$

$T$ is updated in each iteration. When $T$ does not change in two consecutive iterations, then iterations are terminated.

(iv). Choose

$$
G T_{x}(t, \omega)=\left\{\begin{array}{rrr}
0 & \text { if } & G T_{x}(t, \omega) \leq T \\
1 & \text { otherwise }
\end{array} .\right.
$$

Step 3. Classification of auto-components by support vector machine (SVM) of the process performed in step $2[15,30]$. The location and slope of the classification line will determine two important parameters (cut-off lines and rotation order) for filtering in FRFT domain $[15,17]$.

Step 4. Isolation of auto-components in FRFT domain, using step 3 [14, 15].

Step 5. Compute GT and WD of isolated auto-components, using step 4.

Step 6. Compute GWT using the following relation.

$$
\begin{gathered}
G W T_{x}(t, \omega)=G T_{x}^{0.5}(t, \omega) W D_{x}(t, \omega) \\
T F R_{x}(t, \omega)=\sum_{k} G W T_{x_{k}}(t, \omega) \text { where } k=\text { noof auto-components } .
\end{gathered}
$$

\subsection{Numerical simulations}

To show the strength of modified Fractional GWT two examples are considered, (i) three quadratic components and a Gaussian atom, (ii) amplitude varying bat signal [23]. In both examples, auto-components overlap in frequency or in time, and they are also buried in interferences. Thus, these particular case studies show the challenging task to isolate autocomponents from cross-terms in case of WD. 


\subsubsection{Example 1}

$$
\begin{aligned}
x(t)= & 0.2 \exp \left(-2 \pi j\left(7 t^{3}+55 t\right)\right)+0.5 \exp \left(-2 \pi j\left(7 t^{3}+35 t\right)\right)+ \\
& 0.9 \exp \left(-2 \pi j\left(7 t^{3}+15 t\right)\right)+0.7 \exp (-2 \pi j(70 t)) \exp \left(-15 t^{2}\right) .
\end{aligned}
$$

Consider (28), the example of a three quadratic components with time-varying amplitude and a Gaussian atom as shown in Fig. 1 (sampling frequency $=200 \mathrm{~Hz}$, time duration $=-1$ to 1 seconds). The quadratic nature of WD produces cross-terms (Fig. 1 a). Analysis of this signal through GT shows that it provides cross-term elimination property of GT but autocomponents are blurred (Fig. 1 b). The behavior of different variants of GWT (8, 9, 10, and 11) represent issues of readability and missing of auto-components (Fig. $1 \mathrm{c}, \mathrm{d}, \mathrm{e}, \mathrm{f}$ ). By applying the proposed algorithm step by step it proved itself as a powerful tool for analysis of a multi-component signal (Fig. $1 \mathrm{~g}$ ). It provides cross-term elimination property of GT and high resolution property of WD. It extracts successfully all auto-components and gives highly readable TFR. This example also proves that the marriage of signal processing and image processing techniques successfully removes the cross- terms of WD and gives high resolution TFR (as shown in Table 1 and 2).
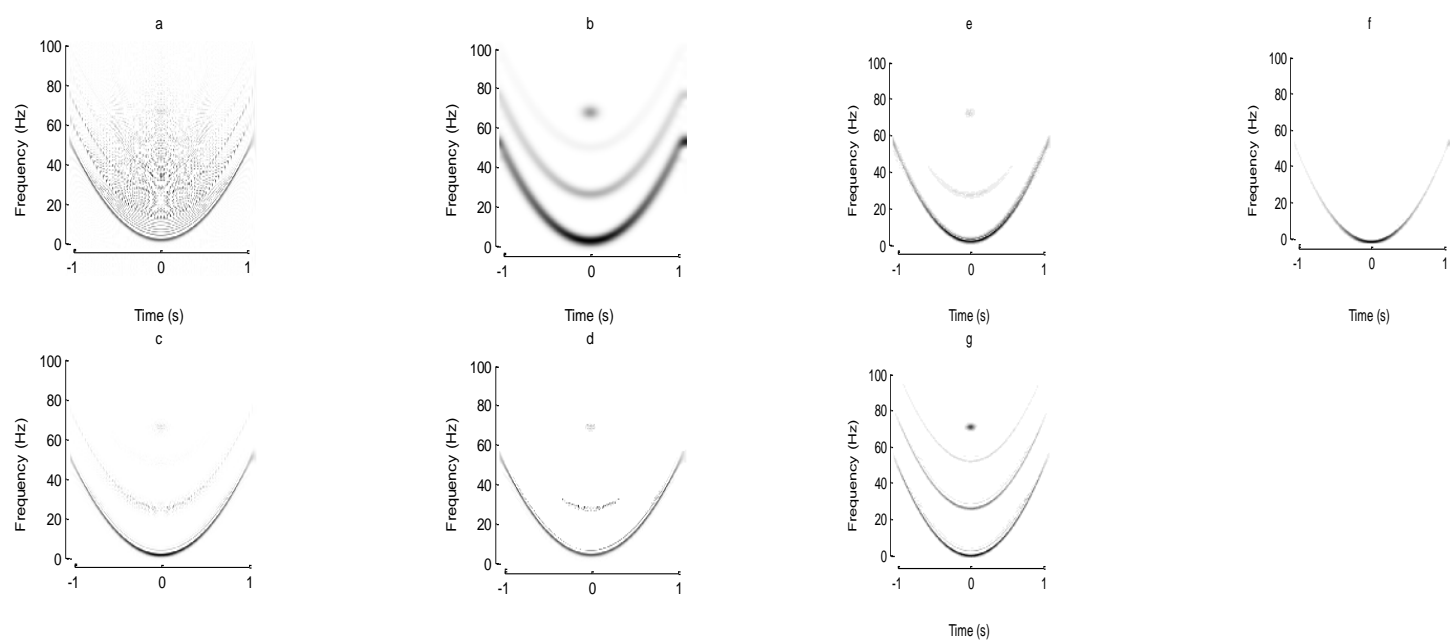

Fig. 1. Analysis of a three quadratic and a Gaussian atom (a) WD, (b) GT, (c) GWT (Eq. 8), (d) GWT (Eq. 9), (e) GWT (Eq. 10), (f) GWT (Eq. 11) and (g) Modified Fractional GWT.
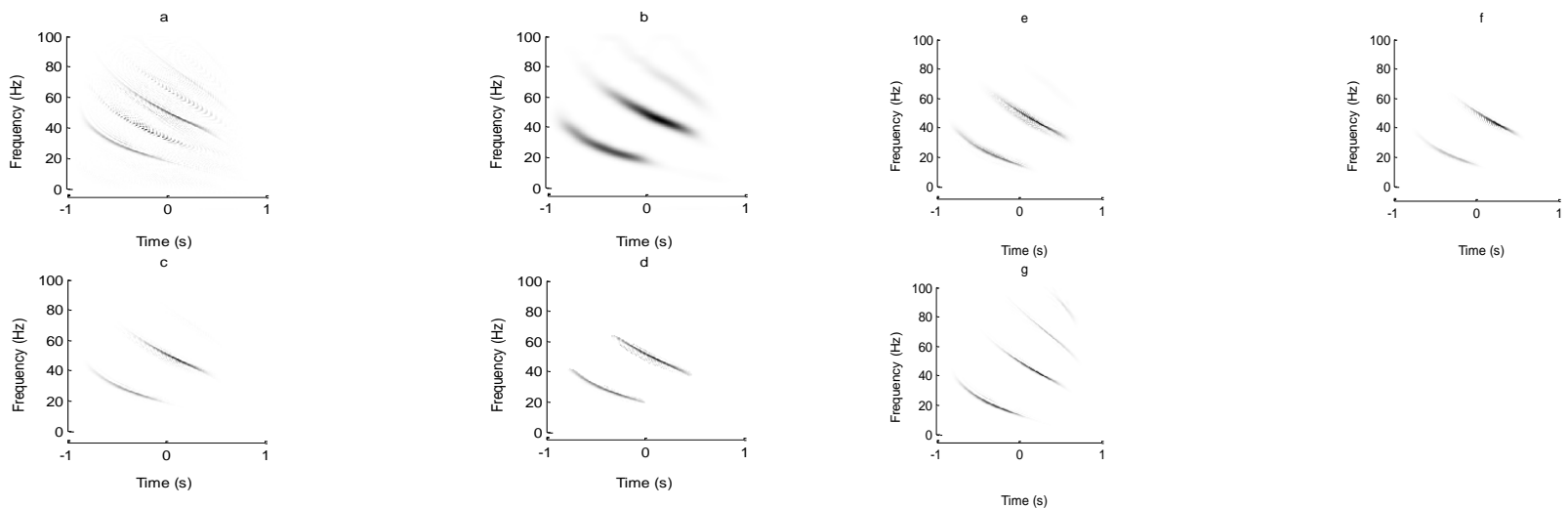

Fig. 2. Analysis of a bat signal (a) WD, (b) GT, (c) GWT (Eq. 8), (d) GWT (Eq. 9), (e) GWT (Eq. 10), (f) GWT (Eq. 11) and (g) Modified Fractional GWT. 


\subsubsection{Example 2}

Consider the example of a real life bat signal [31]. This signal is commonly used for comparison of TFRs. Analysis of the bat signal through the proposed fractional GWT shows that it provides the cross-term elimination property of GT and high resolution property of WD and extracts all auto-components (Fig. 2 g), whereas variants of GWT (Fig. 2 c, d, e, f) miss two auto-components. GT suffers from blurring (Fig. 2 b).

\section{Performance Analysis}

In literature TFRs are compared on the basis of their readability, cross-terms suppression, resolution and energy concentration. Cross-terms suppression and energy concentration of a TFR is evaluated by visual inspection or on the basis of quantitative measures like entropy measures and ratio of norms.

Entropy is used to compute information. Renyi entropy [32] of a TFR $(Q(n, \omega))$ is defined as:

$$
\text { Entropy }_{\operatorname{Re} n y i}=\frac{1}{1-\alpha} \log _{2}\left(\sum_{n} \sum_{\omega} Q^{\alpha}(n, \omega)\right),
$$

where $\alpha$ is the order of entropy. We have taken its value to be equal to 3 , in order to make the above mentioned measure energy unbiased. In case of TFRs, high entropy means lower energy concentration of auto-components; whereas low entropy stands for high concentration of auto-components.

Ratio of norms divides the fourth power norm of a TFR $(Q(n, \omega))$ by its second power norm [33]. Mathematically

$$
\text { Ratio of norms }=\frac{\sum_{n} \sum_{\omega}|Q(n, \omega)|^{4}}{\left(\sum_{n} \sum_{\omega}|Q(n, \omega)|^{2}\right)^{2}} .
$$

Higher value of ratio of norms implies that signal auto-components are highly concentrated [33].

Table 1. Comparison of modified fractional GWT with other TFRs based on ratio of norms.

\begin{tabular}{|l|l|l|l|l|l|l|l|l|}
\hline \multicolumn{7}{|c|}{ Performance measure on the basis of Ratio of Norms $\left(\mathrm{x} 10^{-3}\right)$} \\
\hline S.No. & Test signal & WD & GT & GWT (Eq.8) & GWT (Eq.9) & GWT (Eq.10) & $\begin{array}{l}\text { GWT } \\
\text { (Eq.11) }\end{array}$ & $\begin{array}{l}\text { Modified } \\
\text { Fractional } \\
\text { GWT }\end{array}$ \\
\hline 1 & $\begin{array}{l}3 \text { quadratic } \\
\text { components and } \\
\text { a Gaussian atom }\end{array}$ & 0.0261 & 0.0219 & 0.1599 & 0.0835 & 0.0849 & 0.1849 & 0.2489 \\
\hline 2 & Bat signal & 0.4 & 0.2 & 1.5 & 0.8 & 0.9 & 1.4 & 1.6 \\
\hline
\end{tabular}

The performance of the proposed method is evaluated on the basis of quantitative measures like entropy measures and ratio of norms. The proposed method has a maximum value for ratio of norms for both signals as shown in Table 1 and minimum value of entropy as shown in Table 2. Therefore the proposed GWT shows good energy concentration property as 
compared to other TFRs. Hence our analysis shows that the proposed GWT provides advantages of both WD and GT and also gives the solution of cross-terms of WD.

Table 2. Comparison of modified fractional GWT with other TFRs based on entropy.

\begin{tabular}{|l|l|l|l|l|l|l|l|l|}
\hline \multicolumn{9}{|c|}{ Performance measure on the basis of Entropy } \\
\hline S.No. & Test signal & WD & GT & $\begin{array}{l}\text { GWT } \\
\text { (Eq.8) }\end{array}$ & GWT (Eq.9) & GWT(Eq.10) & GWT(Eq.11) & $\begin{array}{l}\text { Modified } \\
\text { Fractional } \\
\text { GWT }\end{array}$ \\
\hline 1 & $\begin{array}{l}3 \text { quadratic } \\
\text { components and } \\
\text { a Gaussian atom }\end{array}$ & 16.2640 & 16.1286 & 14.2950 & 14.5171 & 14.8538 & 15.8530 & 13.5344 \\
\hline 2 & Bat signal & 13.2170 & 13.6101 & 10.7382 & 11.1182 & 11.3310 & 12.2310 & 10.3854 \\
\hline
\end{tabular}

\section{Conclusion}

In this paper, the advantages of GWT are analyzed in the FRFT domain for multicomponent signals. In our proposed technique, we introduce a novel strategy to eliminate cross terms with minimal distortion in the auto-components through the FRFT domain. The marriage of signal processing and image processing techniques successfully removes the cross- terms of WD and gives high resolution TFR. Performance analysis of the proposed method reveals that it provides the solution of cross-terms of WD and resolution problem of GT and defines further direction of research in this topic.

\section{References}

[1] Cohen, L. (1995). Time-Frequency Analysis. Prentice Hall, Englewood Cliffs, NJ.

[2] Lusin, T., Agrez, D. (2011). Estimation of the amplitude square using the interpolated DFT. Metrol. Meas. Syst., 18(4), 583-596.

[3] Hlawatch, F., Boudreaux-Bartels, G.F. (1992). Linear and quadratic time frequency signal representations. IEEE Signal Processing Magazine, 9(4), 21-67.

[4] Shafi, I., Ahmad, J., Shah, S.I., Kashif, F.M. (2009). Techniques to Obtain Good Resolution and Concentrated Time-Frequency Distributions: A Review. EURASIP Journal on Advances in Signal processing. Artcle ID 673539, doi: 10.1155/2009/673539.

[5] Boashash, B. (2003). Time-Frequency Signal Analysis and Processing. Prentice-Hall, Upper Saddle River, NJ, USA.

[6] Cohen, L. (1989). Time-frequency distributions-A review. In Proc. IEEE, 77, 941-981.

[7] Classen, T.A.C.M., Mecklenbrauker, W.F.G. (1980). The Wigner distribution - a tool for time-frequency signal analysis, part I: Continuous time signals. Philips J. Res., 35, 217-250.

[8] Gröchenig, K. (2001). Foundations of Time-Frequency Analysis. Birkhäuser, Boston.

[9] Lerga, J., Sucic, V., Boashash, B. (2011). An Efficient Algorithm for Instantaneous Frequency Estimation of Nonstationary Multicomponent Signals in Low SNR. EURASIP J. on Adv. In Signal processing, Artcle ID 725189, doi: 10.1155/2011/725189.

[10] Hlawatsch, F., Flandrin, P. (1997). The interference structure of the Wigner distribution and related time-frequency signal representations, in The Wigner Distribution-Theory and Applications in Signal Processing, Elsevier, Amsterdam, Netherlands, 59-133.

[11] Qian, S. (2002). Introduction to Time-Frequency and Wavelet Transforms. Upper Saddle River, New Jersey, Prentice-Hall. 
[12] Hlawatsch, F., Manickam, T.G., Urbanke, R.L., Jones, W. (1995). Smoothed pseudo-Wigner distribution, choi-williams distribution, and cone-kernel representation: Ambiguity-domain analysis and experimental comparison. Signal Process., 43(2).

[13] Arce, G.R., Hasan, S.R. (2000). Elimination of interference terms of the discrete Wigner distribution using nonlinear filtering. IEEE Trans. Signal Processing, 48(8), 2321-2331.

[14] Qazi, S., Georgakis, A., Stergioulas, L.K., Bahaei, M.S. (2007). Interference suppression in the Wigner distribution using fractional Fourier transformation and signal synthesis. IEEE Trans. Signal Process., $55,3150-3154$.

[15] Khan, N.A., Taj, I.A., Jaffri, N., Ijaz, S. (2011). Cross-term elimination in Wigner distribution based on 2D signal processing techniques. Signal Processing, Advances in Fractional Signals and Systems, 91(3), 590-599.

[16] Sejdic, E., Djurovic, I., Stankovic, L. (2011). Fractional Fourier transform as a signal processing tool: An overview of recent developments. Signal Processing, 91, 1351-1369.

[17] Pei, S.C., Ding, J.J. (2007). Relations between Gabor transforms and fractional Fourier transforms and their applications for signal processing. IEEE Trans. Signal Processing, 55(10), 4839-4850.

[18] Cho, S.H., Jang, G., Kwon, S.H. (2010). Time-Frequency Analysis of Power-Quality Disturbances via the Gabor-Wigner Transform. IEEE Trans. Power Deliver., 25(1), 494-499.

[19] Szmajda, M., Górecki, K., Mroczka, J. (2010). Gabor Transform, SPWVD, Gabor-Wigner Transform and Wavelet Transform - Tools for Power Quality monitoring. Metrol. Meas. Syst., 17(3), 383-396.

[20] Rioul, O., Vetterli, M. (1991). Wavelets and Signal Processing. IEEE SP Magazine, 8, 14-38.

[21] Allen, R.L., Mills, D.W. (2004). Signal Analysis: Time, Frequency, Scale, and Structure. New York: Wiley-Interscience.

[22] Hlawatsch, F. (1984). Interference terms in the Wigner distribution. In Proc. Int. Conf. on Digital Signal Processing, Florence, Italy, 363-367.

[23] Stanković, L.J., Alieva, T., Bastiaans, M.J. (2003). Time-frequency signal analysis based on the windowed fractional Fourier transform. Signal Process., 83(11), 2459-2468.

[24] Namias, V. (1980). The fractional order Fourier transform and its applications to quantum mechanics. J. Inst. Math Appl., 25, 241-265.

[25] Mendlovic, D., Ozaktas, H.M. (1993). Fractional Fourier transforms and their optical implementation. J. Opt. Soc. Am. A, 10, 1875-1881.

[26] Kutay, M.A., Ozaktas, H.M., Arikan, O., Onural, L. (1997). Optimal filter in fractional Fourier domains. IEEE Trans. Signal Process., 45(5), 1129-1143.

[27] Almeida, L.B. (1994). The fractional Fourier transform and time-frequency representations. IEEE Trans. Signal Process., 42, 3084-3091.

[28] Saxena, R., Sing, K. (2005). Fractional Fourier transform: A novel tool for signal processing. J. Indian Institute of Science., 85, 11-26.

[29] Acharya, T., Ray, A. (2005). Image Processing: Principles and Applications. Wiley Interscience, Hoboken, NJ.

[30] Training a support vector machine in the primal, Neural Computation. (2007). 19(5), 1155-1178.

[31] /http://www.dsp.rice.edu/software/TFA/RGK/BAT/batsig.bin.ZS.

[32] Williams, W.J., Brown, M., Hero, A. (1991). Uncertainty, information and time frequency distributions. In SPIE, Advanced Signal Processing Algorithms, 1556, 144-156.

[33] Jones, D., Park, T., (1992). A resolution comparison of several time-frequency representations. IEEE Trans. Signal Process., 40, 413-420. 